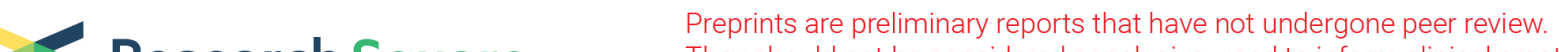 Research Square They should not be considered conclusive, used to inform clinical practice, or referenced by the media as validated information.
}

\section{Prognostic factors of disease-free survival in postoperative patients with hepatocellular carcinoma}

panquan luo

Anhui Medical University

lixiang zhang

First Affiliated Hospital of Anhui Medical University

lei chen

Wenzhou Medical University First Affiliated Hospital

gang wang

Anhui Medical University

hai zhu

First Affiliated Hospital of Anhui Medical University

baichuan zhou

First Affiliated Hospital of Anhui Medical University

wenxiu han

First Affiliated Hospital of Anhui Medical University

aman xu ( $\square$ xamlpqdoctor@163.com )

Anhui Medical University

\section{Research}

Keywords: primary hepatocellular carcinoma, DFS, prognosis

Posted Date: August 11th, 2020

DOI: https://doi.org/10.21203/rs.3.rs-56060/v1

License: (1) (1) This work is licensed under a Creative Commons Attribution 4.0 International License. Read Full License 


\section{Abstract}

Background Hepatocellular carcinoma (HCC) is a primary malignancy of the hepatocyte with high risk of invasion, metastasis and poor prognosis, which leads to a high mortality rate in recent years. This study aims to investigate the independent prognosis factors of DFS(disease free survival) in hepatocellular carcinoma patients after radical hepatectomy.

Methods This study retrospectively analyzed 358 patients admitted to the 1st Affiliated Hospital of Anhui Medical College from December 2009 to December 2014. Categorical variables were analyzed by chisquare test. The survival curve was plotted by kaplan-meier method and tested by log-rank method. Univariate and multivariate Cox regression analyses were performed to determine the prognostic factors.

Results Among the 344 patients with liver cancer, 298 cases (86.63\%) are young patients ( $<70$ years), and 46 cases $(13.37 \%$ ) were elderly patients ( $\geq 70$ years). There were no significant difference in gender, smoking history, alcohol consumption history, Body Mass Index (BMI), number of cancer nodules, tumor diameter, differentiation degree and TNM stage between the young and elderly groups $(P>0.05)$. However, history of hepatitis B and liver cirrhosis were more common in young patients than elderly $(P=0.017, P \otimes 0.000)$. The Kaplan-Meier curves indicated that elderly patients (higher than 70 years), TNM III stage and no history of hepatitis B had longer DFS. The univariate and multivariate analyses showed that age, TNM stage, History of hepatitis B were the independent factors for DFS.

Conclusions Our findings suggested that age, TNM stage, history of hepatitis B were independent factors for predicting DFS of primary hepatocellular carcinoma patients. Patients older than 70 years had a better prognosis than young patients. Patients with early TNM stage and no history of hepatitis B had longer DFS.

\section{Introduction}

On the basis of global cancer statistics, primary liver cancer ranks fifth among all types of cancer, and ranks fourth in cancer-related death worldwide. According to recent WHO data, as many as 781,631 people die of liver cancer every year in the world ${ }^{1,2}$. Primary liver cancer include hepatocellular carcinoma(HCC), intrahepatic cholangiocarcinoma(ICC) and hepatocholangiocarcinoma(cHCC-ICC) according to histological classification. Approximately $70-90 \%$ of primary liver cancer are hepatocellular carcinoma (HCC) ${ }^{3}$.

With helical computed tomography (CT), magnetic resonance imaging (MRI) examination and the level of treatment methods have been greatly improved, patients with early liver cancer underwent radical surgical resection, the 5-year survival rate can reach $70 \%{ }^{4}$. However, Most patients with liver cancer who come to the hospital due to dull pain in the upper abdomen or other symptoms are already in middle or later stage, the 5-year survival rate is less than $15 \%{ }^{5}$. Therefore, it is urgent to study the prognostic factors of HCC. 
The factors and pathogenesis of hepatocellular carcinoma are controversial comprising of multiple genetic, epigenetic alterations, chromosomal aberrations and gene mutations ${ }^{6}$. The process of tumor development is complicated. It has been reported that the occurrence of liver cancer is related to viral hepatitis, especially hepatitis $B^{7}$. At present, radical Hepatectomy is the first choice for the treatment of hepatocellular carcinoma patients. After the operation, the necessary and appropriate chemotherapy regimen should be given ${ }^{8}$. Tumor recurrence was determined mainly based on the imaging results of postoperative follow-up or the re-pathological results of the patients, and DFS was calculated, that is the time from the disease progression or the last follow-up to the date of surgery, which is a favorable indicator to observe the postoperative prognosis of cancer ${ }^{9}$.

Up to date,many clinical studies have been performed to identify the corresponding markers that can accurately predict the prognosis of liver cancer. Unfortunately, no clear prognostic factors for hepatocellular carcinoma patients have been identified. A great deal of clinical investigations indicated that factors affecting overall survival of HCC include age, HBsAg, alcohol consumption history, TNM stage, differentiation degree, tumor number, tumor size, AFP, GGT, NLR and so on ${ }^{10-13}$.

Jun Zheng et al. ${ }^{14}$ suggested NLR and PLR, which were SIR (Systemic inflammatory response) indexes, were new prognostic markers for predicting the prognosis of HCC. Increased NLR or PLR was found to be an independent predictor of higher recurrence and poor survival in HCC patients receiving curative or palliative therapy. Brian K. P. Goh et al. ${ }^{15}$ showed number of cancer nodules (more than three nodules), Child-Pugh status, margin positivity and presence of microvascular invasion, but not tumor size, were independent negative predictors of OS (overall survival). In addition, in the retrospective study of 414 patients with liver cancer over a period of 7 years, our team found that serum amma-glutamyltransferase levels and AST/ALT play an important role in the prediction of OS in patients with primary liver cancer. Low GGT group and low AST/ALT group have a better prognosis ${ }^{16}$. Our group has studied the prognostic factors of OS in patients with liver cancer, but no further study has been conducted on DFS in liver cancer patients, especially hepatocellular carcinoma patients.

The purpose of this study was to explore the clinical factors affecting DFS in patients with HCC after radical resection, so as to take appropriate therapeutic measures for maximizing these patients DFS.

\section{Methods}

\section{Patients}

This study retrospectively recruited 344 cases of hepatocellular carcinoma patients that received curative hepatectomy during the Department of Gastrointestinal Surgery,the first Affiliated Hospital of Anhui Medical College. The inclusion criteria were: 1) All patients with HCC were confirmed histo-pathologically; 2) Resection of liver cancer was necessary; 3 ) The clinical data of the patients were complete and correct; 4) The survival of patients could be followed up; 5) no other treatment, such as chemoradiotherapy and interventional therapy before surgery. Exclusion of patients criteria were: 1) Postoperative pathology: bile 
duct cell carcinoma or metastatic liver cancer; 2) Incomplete data; 3) Child-pugh grading of liver function was grade $\mathrm{C}$; 4 ) Inability to follow up or loss of follow-up. We collected information of patients through the record room of our hospital, including patients gender, age, personal history (smoking history, alcohol use history), BMI, presence of basic diseases (hypertension, diabetes, history of hepatitis B), presence of liver cirrhosis, tumor number, tumor size, differentiation degree, TNM stage, number of cancer nodules, AFP, portal vein thrombosis, ascites, routine peripheral blood examination (neutrophils, lymphocytes and platelets) and anticoagulation test (prothrombin time, fibrinogen), liver function test (ALB(albuminbilirubin), TBIL(total bilirubin), TC(total cholesterol), ALT(alanine aminotransferase), AST(aspartate aminotransferase), GGT(gamma-gl utamyltransferase)). In our study, the patients were divided into a High group and normal group based on their thresholds levels of serum ALB, TBIL, TC, TG, ALT, AST and GGT. In addition, the median of NLR and PLR were used as cutoff value, respectively. This study was approved by the ethics committee of the 1st Affiliated Hospital of Anhui Medical College, and all patients signed the preoperative informed consent.

\section{Follow-up And Treatment}

The follow - up information of the patients was obtained through telephones and outpatient visit. The follow - ups carried out in normal intervals (follow-up began one month after the surgery, each 3 months within two years, and each 6 months from the third years to fifth year, and once a year after the fifth year). Routine peripheral blood examination, liver function test, liver ultrasound or CT were performed at each follow-up patient after hepatectomy.

\section{Statistical analysis}

All patient data were statistically described using spss 19.0 software. Chi-square test or Fisher exact test were used to investigate categorical variables. Univariate analysis was performed for all variables, and then the variables of $\mathrm{P}<0.05$ were selected for multivariate Cox regression analysis. The kaplan-meier method was performed to analyze DFS and survival curves were compared using the log-rank test in each group. Cox proportional hazards regression were utilized to assess the relationship between different groups and DFS in patients. The hazard ratio (HR) and $95 \%$ confidence interval $(95 \% \mathrm{Cl})$ were used to show the risks. $H R>1.0$ showed an increased risk of death. $P$ value $<0.05$ was considered statistically significant.

\section{Results}

\section{Clinical characteristics of patients}

A total of 344 patients with hepatocellular carcinoma were included in this study, among whom 279 cases were males and 65 cases were females, accounting for $81.10 \%$ and $18.90 \%$ respectively. Details of patients' information were shown in table 1 . The patients were divided into two groups according to their 
age, namely the elderly group with age $>70$ years old and the young group with age $<70$ years old. Consequently, there were 298 patients in young group, with a median age of 55.00 (inter-quartile range: 47.00-61.00) years old and 74.00 (inter-quartile range:72.00-77.00) years in the elderly group. The number of patients who had smoking history reached 151 , accounting for $43.90 \%$. And $133(38.66 \%)$ patients had alcohol use history. While $258(75 \%)$ patients had a history of hepatitis B, only $86(25 \%)$ cases did not. Most patients had no history of hypertension or diabetes $(76.74 \%$ and $88.08 \%)$. Nearly half of the patients $(45.93 \%)$ had a BMI more than 23. Moreover, the patients who had liver cirrhosis were 256 (74.42\%), 88 patients without liver cirrhosis, nevertheless, most of them had abnormal liver function, such as hepatitis and fatty liver. $44(12.79 \%)$ cases had ascites, unfortunately, two of them had bloody ascites. There were 181 patients with tumor diameter less than $5 \mathrm{~cm}$, which was slightly higher than the number of tumor diameter $\geq 5 \mathrm{~cm}(52.62 \%$ vs. $47.38 \%)$. The pathology differentiation grade of 259 cases were moderate and high, and only a small number of patients (85 cases) were poorly differentiated. The TNM stage of I-II and III-IV were 300 (87.21) cases and 44 (12.79\%) cases. The median of NLR and PLR was 2.19 (inter-quartile range: 1.64-3.31) and 97.67 (72.10-138.17), respectively. The median follow-up time for this study was 52.00 (10.00-78.75) months. During the follow-up period, the 1-year, 3-year and 5-year DFS of the patients were $73.26 \%, 59.30 \%$ and $44.48 \%$, respectively.

\section{Correlation between different age groups and clinicopathological characteristics of patients}

The results of the correlation between clinical and pathological indicators in the elderly group and the young group were shown in Table 2 . The association between age and history of hepatitis $B$, hypertension, diabetes and liver cirrhosis were significant $(P<0.05)$. Furthermore, the history of hepatitis $B$ and liver cirrhosis in the young group were significantly higher than that in the elderly group $(77.18 \%$ vs. $60.87 \%$ and $77.85 \%$ vs. $52.17 \%$ ). Patients without hypertension and diabetes appeared to be less common in elderly patients than in the young group ( $45.65 \%$ vs. $81.54 \%$ and $80.43 \%$ vs. $89.26 \%$ ). As for hematological index, the age was significantly connected with HbsAg, AST, ALB, PLR $(P<0.05)$. However, there were no statistical differences between the two groups with regard to smoking, alchohol intake, BMI, tumor number, tumor diameter, differentiation degree, TNM stage, AFP, TBIL, TC, ALT, GGT and NLR.

\section{The Prognostic Factors For DFS}

The results of univariate regression analysis were expressed in Table 3. Age, history of hepatitis B, hypertension, number of tumor nodules, tumor diameter, TNM stage, and portal vein embolization were correlated with patients DFS $(P<0.05)$. Table 4 showed the multivariate analysis of DFS which included the significant factors obtained from Table 3. Multivariable Cox regression analysis revealed that age, history of hepatitis $B$ and TNM stage were independent factors for predicting disease-free survival rate of primary Hepatocellular carcinoma patients. Besides, the hazard rate (HR) was 0.543 (95\% Cl:0.328- 
0.898) for patients older than 70, $0.654(95 \% \mathrm{Cl}: 0.472-0.907)$ for patients without history of hepatitis $\mathrm{B}$, and $0.585(95 \% \mathrm{Cl}: 0.423-0.810)$ for patients with TNM stage of I-II.

\section{Results of DFS in different age groups, AJCC stage and with or without history of hepatitis B}

As shown in Fig. 1,the DFS of the different age groups were compared with Kaplan-Meier plot. The results indicated that patients with age $\geq 70$ have a longer DFS $(P<0.05)$. As can be seen from the Kaplan-Meier curves, the survival rate in the young group showed the most significant downward trend in the first 6 months of follow-up, suggested that the patients in the young group had a greater risk of recurrence or even death within 6 months after surgery. Figures 2 and 3 showed DFS at different TNM stage and with or without a history of hepatitis B, respectively. From the figures, we found that the median DFS of the stage I-II group was 68 months, and the median DFS of the stage III- IV group was 12 months. patients with TNM stage of $\mathrm{I}-\mathrm{II}$ and no history of hepatitis $\mathrm{B}$ had longer DFS $(\mathrm{P}<0.05)$.

In order to investigate the association between TNM stage and the different age groups further,we performed survival analysis and results were shown in Fig. 4A and Fig. 4B. Figure 4A showed that in TNM stage of I-II, the elderly group possessed a longer DFS than the young group. However, there was no significant difference in TNM stage of III- IV. In addition】we also investigated the association between age and different TNM stage groups through Fig. 5A and Fig. 5B. The results suggested that the young group also had a better prognosis in TNM stage of I-II. In contrast, the difference was not statistically significant in the elderly group. Furthermore, the association between TNM stage and with or without history of hepatitis B was analyzed in Fig. 6A and Fig. 6B and no statistically significance was found.

\section{Discussion}

Primary liver cancer is one of the most common gastrointestinal malignancies. Because there are usually no typical symptoms in the early stage, the diagnosis of some patients is delayed ${ }^{17}$. Hepatocellular carcinoma (HCC) has a high incidence rate and a high mortality rate. As a result, more and more attention has been put to the research of hepatocellular carcinoma prognostic factors ${ }^{18}$.

Our current research retrospectively analyzed the Clinico-pathological information of 344 patients with hepatocellular carcinoma admitted to the first Affiliated Hospital of Anhui Medical College from December 2009 to December 2014. The results of univariate regression analysis indicated that age, history of hepatitis $B$, hypertension, tumor number, tumor diameter, TNM stage, portal vein embolization, and fibrinogen were the prognosis factors of DFS in patients with HCC, and then we performed a multivariate Cox regression analysis of these factors. The results showed that age, history of hepatitis $B$ and TNM stage were independent factors $(P<0.05)$. NLR was calculated using the formula absolute neutrophil count (number of neutrophils $/ \mathrm{ml}$ ) divided by absolute lymphocyte count (number of lymphocytes/ml), PLR was calculated as absolute platelet count (number of platelets/ml) divided by 
absolute lymphocyte count (number of lymphocytes $/ \mathrm{ml}$ ) ${ }^{19}$. Many studies had shown that NLR, AFP and fibrinogen were related to liver cancer patients $0 S^{20,21}$. In our study, we used the median of NLR and PLR as their cutoff values, the values were similar to that previously reported ${ }^{22,23}$. To our surprise, hematological indicators such as NLR, PLR and AFP were not prognosis factors of patients DFS. It demonstrates that the levels of NLR, PLR and AFP are reliable indicators for predicting the OS of patients with liver cancer. However, these indicators are probably not play a role in hepatocellular carcinoma patients DFS.

In our study, the definition of elderly group in hepatocellular carcinoma patients was age of 70 or over based on previous published studies ${ }^{24,25}$. Age $\geq 70$ patients with hepatocellular carcinoma had longer DFS. In order to further understand the influence of age on patients DFS under different AJCC stages, we analyzed the DFS of patients in different age groups under stages I-II and III- IV, respectively. Patients aged $\geq 70$ at stage $\mathrm{I}-\mathrm{Il}$ also had a better prognosis compared to the young group. Interestingly,in study of Atsuko Sakakibara et al. ${ }^{26}$ they divided the age groups into the young group and the old group at the age of 40Пit was observed that despite the older adult group having a higher proportion of advanced cancer patients, the overall survival rate of stage IIB patients in the young group at the 3-year follow-up was significantly lower, This result was similar to what we found in this study. Beside!The similar results were also found in the study of Liying Zhao et al. ${ }^{27}$, this retrospective cohort study included a total of 995 patients with CRC suggested the patients aged 35 years and younger have specific clinicopathological characteristics that tend to a worse prognosis. On the contrary, W. Faber et al. ${ }^{28}$ retrospectively studied 141 patients with liver cirrhosis after curative resection clinical characteristics, and found out prognosis of patients less than 70 years old was significantly better. The 5 -year survival rate reached about $50 \%$, which was significantly higher than that of the elderly group. The most likely reason for these two completely opposite conclusions is that different researchers have different research priorities and parameter Settings, resulting in different results. Therefore, it is necessary to further study the mechanisms by which age affects cancer prognosis and the relationship between age of surgery and DFS in patients with hepatocellular carcinoma. It is generally known that the stage of tumor can greatly affect the prognosis of patients, the more advanced the stage, the worse the prognosis. In this study, we found that regardless of the patients age, the prognosis of patients of TNM stage I-II was significantly better than that of patients with stage III- IV. The results were the same as that of a decade-long and population-based Analysis by Lu Wu et al. ${ }^{29}$. For patients at the early stage, radical surgery should firstly be considered $[$ this may prolong the DFS and help prevent metastasis.

As is known to own, hepatitis B virus (HBV) is a leading causing factors of HCC. HBV infection is a global health problem and roughly $30 \%$ of the world's population shows serological evidence of current or past HBV infection, especially in China. China is a big country with hepatitis $B$, although the success of neonatal vaccination since 1992, which had achieved a great reduction ${ }^{30}$, there are still great challenges, HBV infection affects at least 2 billion people worldwide, and 97 million people are HBV carriers and at least 20 million of them still suffer from active or chronic HBV infection in China ${ }^{31}$. Lian Li et al. ${ }^{32}$ suggested the HBV DNA level was an independent prognostic factor for patients with OS and DFS, in 
addition, HBV DNA level of $2000 \mathrm{IU} / \mathrm{ml}$ or greater before operation indicated a poorer prognosis. As for the pathogenesis of liver cancer caused by HBV, on the one hand, HBV virus gene fragments can be inserted directly into the genomes of normal hepatocytes, causing gene mutations that induce normal cells to transform into tumor cells, especially in patients with high HBV-DNA levels. on the other hand, HBx is a protein encoded by HBV with trans-activation activity which can dysregulate cell transcription and proliferation control and sensitize liver cells to carcinogenic factors ${ }^{33}$. Although HbsAg positivity was not associated with DFS in HCC patients in this study, history of hepatitis B was an independent prognostic factor for DFS in patients with HCC. This may emphasize that we should pay attention to the history of hepatitis B when we ask the history of patients with liver cancer in the course of clinical practice. Postoperative recurrence of hepatocellular carcinoma is a mojor factor affecting the survival of patients with hepatocellular carcinoma. Therefore, it is particularly important to follow up for patients with liver cancer after operation.

The main advantage of this study was that the analyzed factors are relatively comprehensive and can effectively reduce residual confounding.

There were several limitations of our study such as the number of patients in the elderly group was small and single-center retrospective design. Hence, Increasing the sample size and replacing the single center with a multi-center prospective study could make the results more convincing and representative.

\section{Conclusions}

In conclusion, we found that age, TNM stage and history of hepatitis B were independent prognostic factors for patients DFS after radical hepatectomy. Furthermore, the patients older than 70 years old had a longer DFS than young patients, and earlier TNM stage and no history of hepatitis B were associated with a better prognosis. This study may imply us that when we make the treatment plan for patients, we should also take these prognostic factors into consideration. Only in this way can we acquire better personalized treatment for HCC patients.

\section{Declarations}

\section{Acknowledgements}

None.

\section{Funding}

National Natural Science Foundation of China(No.81572350toA.M.X.).

\section{Availability of data and materials}

The data used and/or analyzed during the current study were obtained from the Department of Gastrointestinal Surgery, the First Hospital of Anhui University. The data are available from the 
corresponding author on reasonable request.

\section{Authors' Contributions}

PQL collected the clinical information of patients, performed the statistical analysis, and completed the writing of the manuscript; LXZ assisted in collecting the patients' clinical information and writing the manuscript; $\mathrm{CL}$ assisted in collecting the clinical information of patients $\mathrm{HZ}$ and $\mathrm{GW}$ participated in the review of the drafted manuscript.; BCZ participated in revising the statistical methods of the investigation; AMX and WXH designed the main study and critically revised the manuscript. AMX and WXH contributed equally to this article. All authors read and approved the final manuscript.

\section{Ethics approval and consent to participate}

The present study was reviewed and approved by Ethics Committee of The First Hospital of Anhui University (Hefei, China). Informed consent for use of the medical data in this study was obtained from the patients.

\section{Patient consent for publication}

All patients provided consent for the publication of their data and associated images.

\section{Competing interests}

The authors declare that there are no competing interests associated with the manuscript.

\section{References}

1. Bray F, Ferlay J, Soerjomataram I, Siegel RL, Torre LA, Jemal A. Global cancer statistics 2018 : GLOBOCAN estimates of incidence and mortality worldwide for 36 cancers in 185 countries. CA Cancer J Clin. 2018;68(6):394-424.

2. Feng RM, Zong YN, Cao SM, Xu RH. Current cancer situation in China: good or bad news from the 2018 Global Cancer Statistics? Cancer Commun (Lond). 2019;39(1):22.

3. Grandhi MS, Kim AK, Ronnekleiv-Kelly SM, Kamel IR, Ghasebeh MA, Pawlik TM. Hepatocellular carcinoma: From diagnosis to treatment. Surg Oncol. 2016;25(2):74-85.

4. European Association for the Study of the Liver. Electronic address eee, European Association for the Study of the L. EASL Clinical Practice Guidelines: Management of hepatocellular carcinoma. J Hepatol. 2018;69(1):182-236.

5. Ilikhan SU, Bilici M, Sahin H, et al. Assessment of the correlation between serum prolidase and alphafetoprotein levels in patients with hepatocellular carcinoma. World J Gastroenterol. 2015;21(22):6999-7007. 
6. Fransvea E, Paradiso A, Antonaci S, Giannelli G. HCC heterogeneity: molecular pathogenesis and clinical implications. Cell Oncol. 2009;31(3):227-33.

7. Maucort-Boulch D, de Martel C, Franceschi S, Plummer M. Fraction and incidence of liver cancer attributable to hepatitis B and C viruses worldwide. Int J Cancer. 2018;142(12):2471-7.

8. Schlachterman A, Craft WW Jr, Hilgenfeldt E, Mitra A, Cabrera R. Current and future treatments for hepatocellular carcinoma. World J Gastroenterol. 2015;21(28):8478-91.

9. De La Cruz L, Moody AM, Tappy EE, Blankenship SA, Hecht EM. Overall Survival D-F, Survival. Local Recurrence, and Nipple-Areolar Recurrence in the Setting of Nipple-Sparing Mastectomy: A MetaAnalysis and Systematic Review. Ann Surg Oncol. 2015;22(10):3241-9.

10. Bucci L, Garuti F, Camelli V, et al. Comparison between alcohol- and hepatitis C virus-related hepatocellular carcinoma: clinical presentation, treatment and outcome. Aliment Pharmacol Ther. 2016;43(3):385-99.

11. Yamakado K, Miyayama S, Hirota S, et al. Subgrouping of intermediate-stage (BCLC stage B) hepatocellular carcinoma based on tumor number and size and Child-Pugh grade correlated with prognosis after transarterial chemoembolization. Jpn J Radiol. 2014;32(5):260-5.

12. Liao R, Du CY, Gong JP, Luo F. HBV-DNA Load-Related Peritumoral Inflammation and ALBI Scores Predict HBV Associated Hepatocellular Carcinoma Prognosis after Curative Resection. J Oncol. 2018;2018:9289421.

13. Zhang H, Yu X, Xu J, Li J, Zhou Y. Combined hepatocellular-cholangiocarcinoma: An analysis of clinicopathological characteristics after surgery. Medicine (Baltimore). 2019;98(38):e17102.

14. Zheng J, Cai J, Li H, et al. Neutrophil to Lymphocyte Ratio and Platelet to Lymphocyte Ratio as Prognostic Predictors for Hepatocellular Carcinoma Patients with Various Treatments: a MetaAnalysis and Systematic Review. Cell Physiol Biochem. 2017;44(3):967-81.

15. Goh BK, Chow PK, Teo JY, et al. Number of nodules, Child-Pugh status, margin positivity, and microvascular invasion, but not tumor size, are prognostic factors of survival after liver resection for multifocal hepatocellular carcinoma. J Gastrointest Surg. 2014;18(8):1477-85.

16. Zhang LX, Lv Y, Xu AM, Wang HZ. The prognostic significance of serum gamma-glutamyltransferase levels and AST/ALT in primary hepatic carcinoma. BMC Cancer. 2019;19(1):841.

17. Orcutt ST, Anaya DA. Liver Resection and Surgical Strategies for Management of Primary Liver Cancer. Cancer Control. 2018;25(1):1073274817744621.

18. Llovet JM, Zucman-Rossi J, Pikarsky E, et al. Hepatocellular carcinoma. Nat Rev Dis Primers. 2016;2:16018.

19. Diem S, Schmid S, Krapf M, et al. Neutrophil-to-Lymphocyte ratio (NLR) and Platelet-to-Lymphocyte ratio (PLR) as prognostic markers in patients with non-small cell lung cancer (NSCLC) treated with nivolumab. Lung Cancer. 2017;111:176-81.

20. Chan MY, She WH, Dai WC, et al. Prognostic value of preoperative alpha-fetoprotein (AFP) level in patients receiving curative hepatectomy- an analysis of 1,182 patients in Hong Kong. Transl Gastroenterol Hepatol. 2019;4:52. 
21. Dai T, Peng L, Lin G, et al. Preoperative elevated plasma fibrinogen level predicts tumor recurrence and poor prognosis in patients with hepatocellular carcinoma. J Gastrointest Oncol. 2019;10(6):1049-63.

22. Yang YT, Jiang JH, Yang HJ, Wu ZJ, Xiao ZM, Xiang BD. The lymphocyte-to-monocyte ratio is a superior predictor of overall survival compared to established biomarkers in HCC patients undergoing liver resection. Sci Rep. 2018;8(1):2535.

23. Fu H, Zheng J, Cai J, et al. Systemic Immune-Inflammation Index (SII) is Useful to Predict Survival Outcomes in Patients After Liver Transplantation for Hepatocellular Carcinoma within Hangzhou Criteria. Cell Physiol Biochem. 2018;47(1):293-301.

24. Cucchetti A, Sposito $C$, Pinna AD, et al. Effect of age on survival in patients undergoing resection of hepatocellular carcinoma. Br J Surg. 2016;103(2):e93-9.

25. Hsu KF, Yu JC, Yang CW, et al. Long-term outcomes in elderly patients with resectable large hepatocellular carcinoma undergoing hepatectomy. Surg Oncol. 2018;27(3):595-601.

26. Sakakibara A, Matsui K, Katayama T, Higuchi T, Terakawa K, Konishi I. Age-related survival disparity in stage IB and IIB cervical cancer patients. Journal of Obstetrics Gynaecology Research. 2019;45(3):686-94.

27. Zhao L, Bao F, Yan J, et al. Poor prognosis of young patients with colorectal cancer: a retrospective study. Int J Colorectal Dis. 2017;32(8):1147-56.

28. Faber W, Stockmann M, Schirmer C, et al. Significant impact of patient age on outcome after liver resection for HCC in cirrhosis. Eur J Surg Oncol. 2014;40(2):208-13.

29. Wu L, Tsilimigras DI, Farooq A, et al. Management and outcomes among patients with sarcomatoid hepatocellular carcinoma: A population-based analysis. Cancer. 2019;125(21):3767-75.

30. Cui F, Shen L, Li L, et al. Prevention of Chronic Hepatitis B after 3 Decades of Escalating Vaccination Policy, China. Emerg Infect Dis. 2017;23(5):765-72.

31. Wang FS, Fan JG, Zhang Z, Gao B, Wang HY. The global burden of liver disease: the major impact of China. Hepatology. 2014;60(6):2099-108.

32. Li L, Li B, Zhang M. HBV DNA levels impact the prognosis of hepatocellular carcinoma patients with microvascular invasion. Medicine (Baltimore). 2019;98(27):e16308.

33. Levrero M, Zucman-Rossi J. Mechanisms of HBV-induced hepatocellular carcinoma. J Hepatol. 2016;64(1 Suppl):84-101.

\section{Figures}




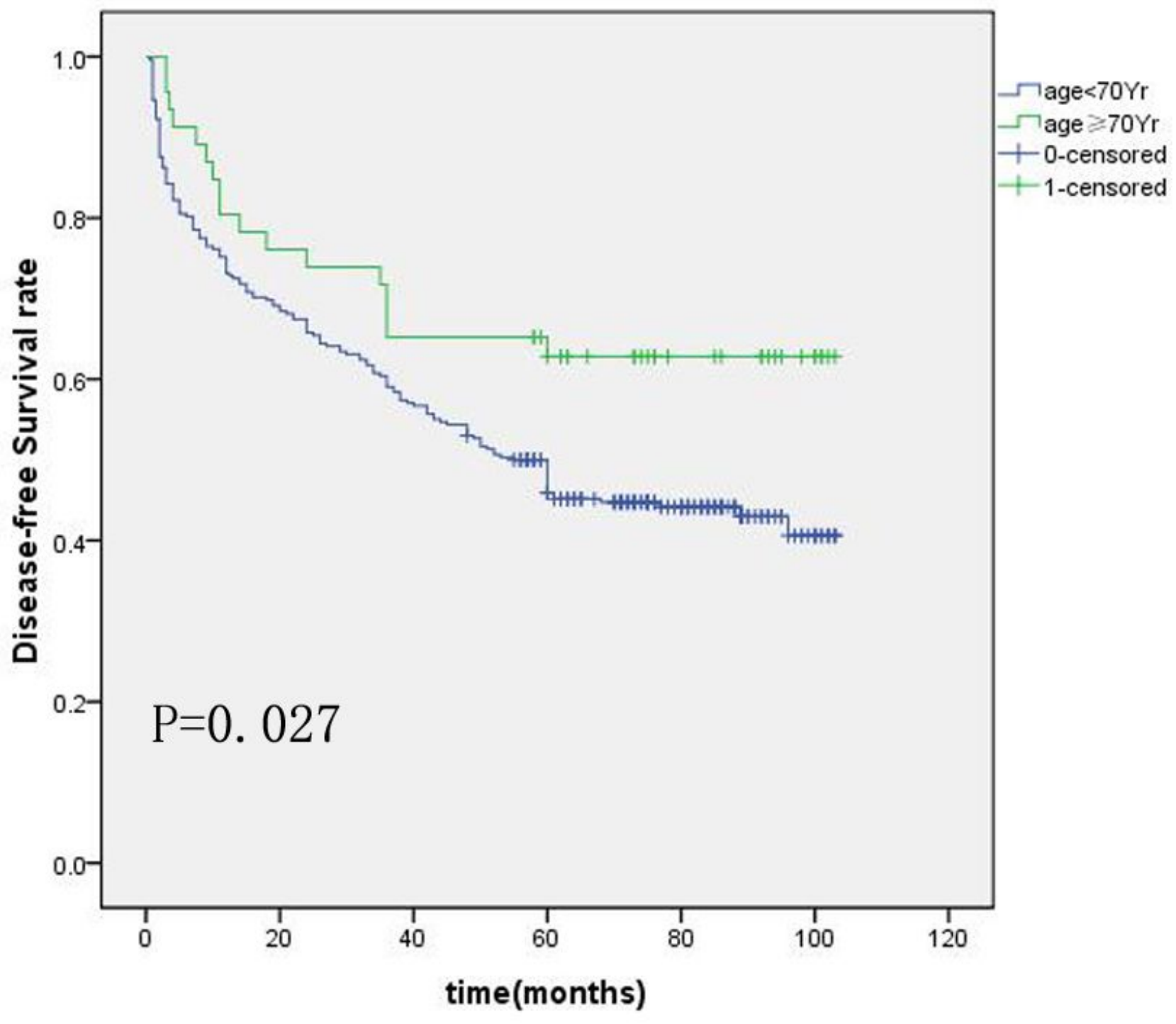

Figure 1

Kaplan-Meier curve for Disease-Free Survival according to age groups. 


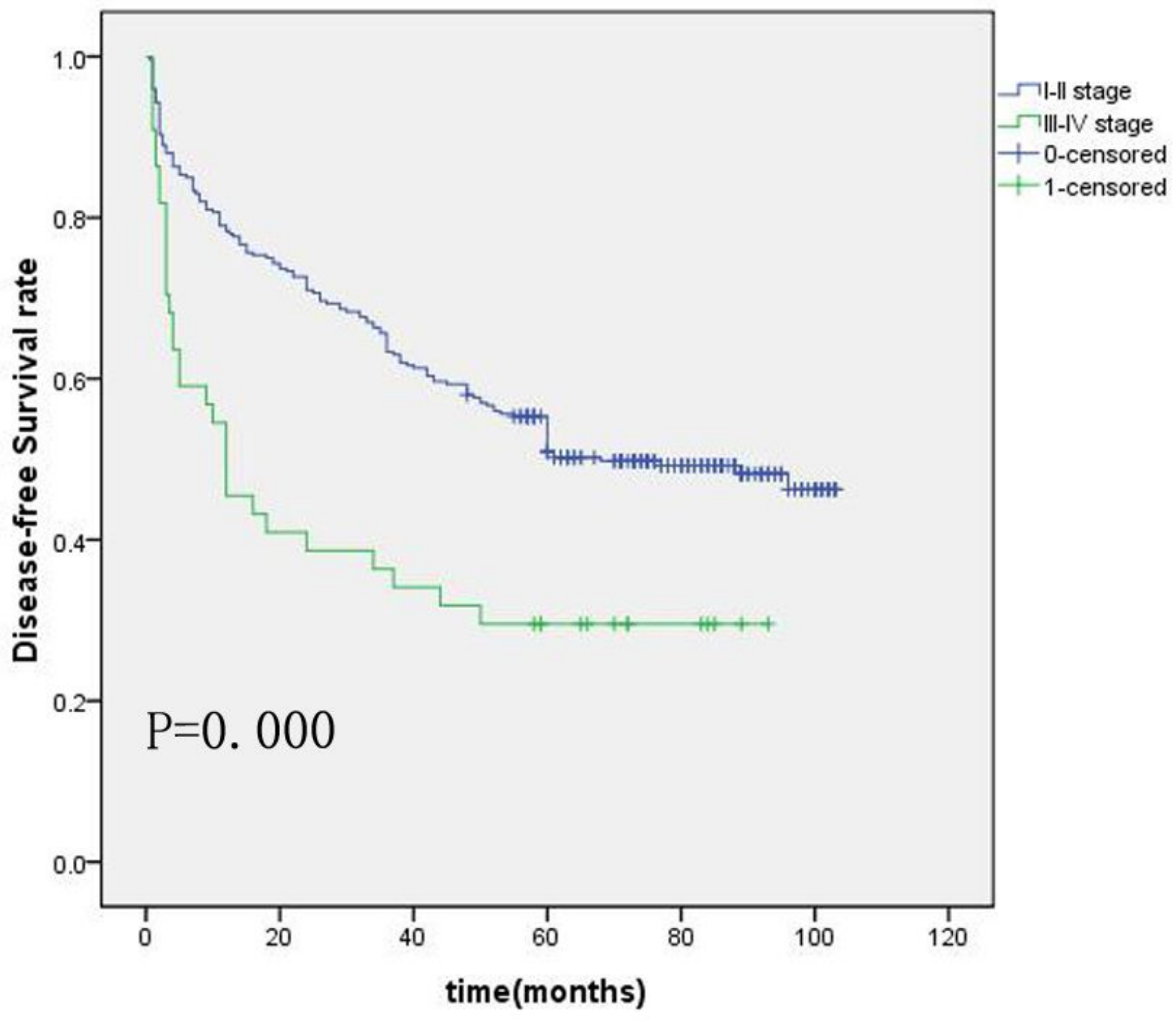

Figure 2

Kaplan-Meier curves for Disease-Free Survival in different age groups based on (A) stage I- II and (B) stage III- IV. 


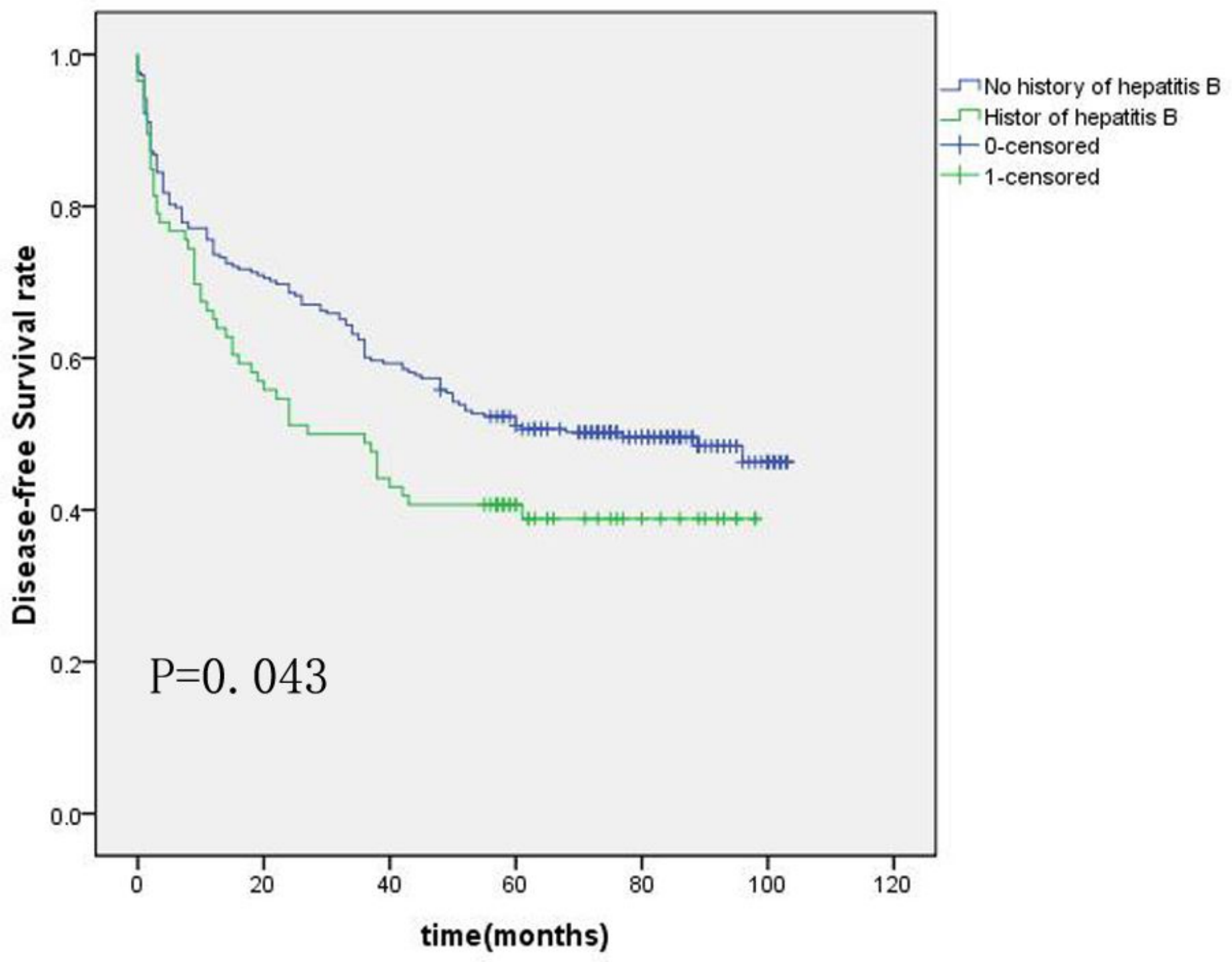

Figure 3

Kaplan-Meier curve for Disease-Free Survival according to stage groups. 

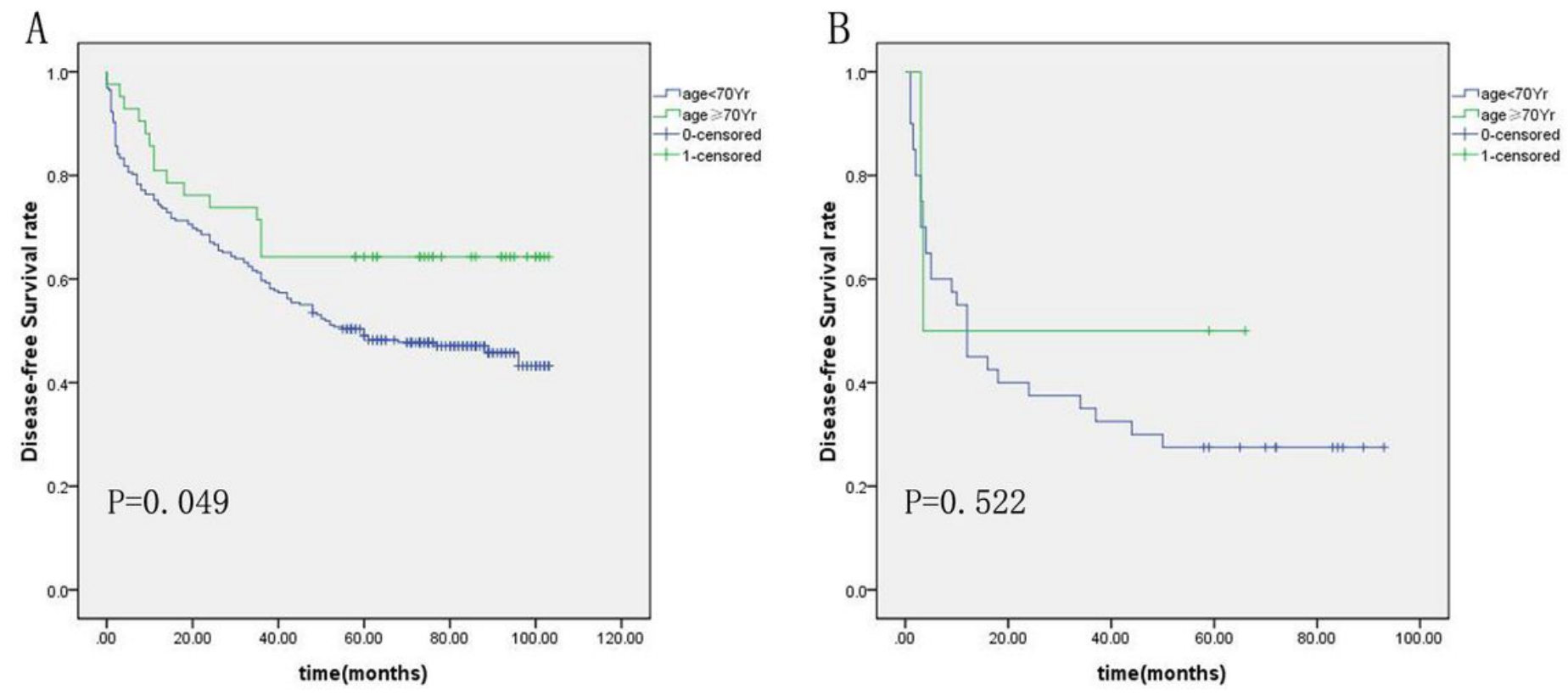

\section{Figure 4}

Kaplan-Meier curves for Disease-Free Survival in different stage groups based on (A) age $\geq 70$ and (B) age $<70$.
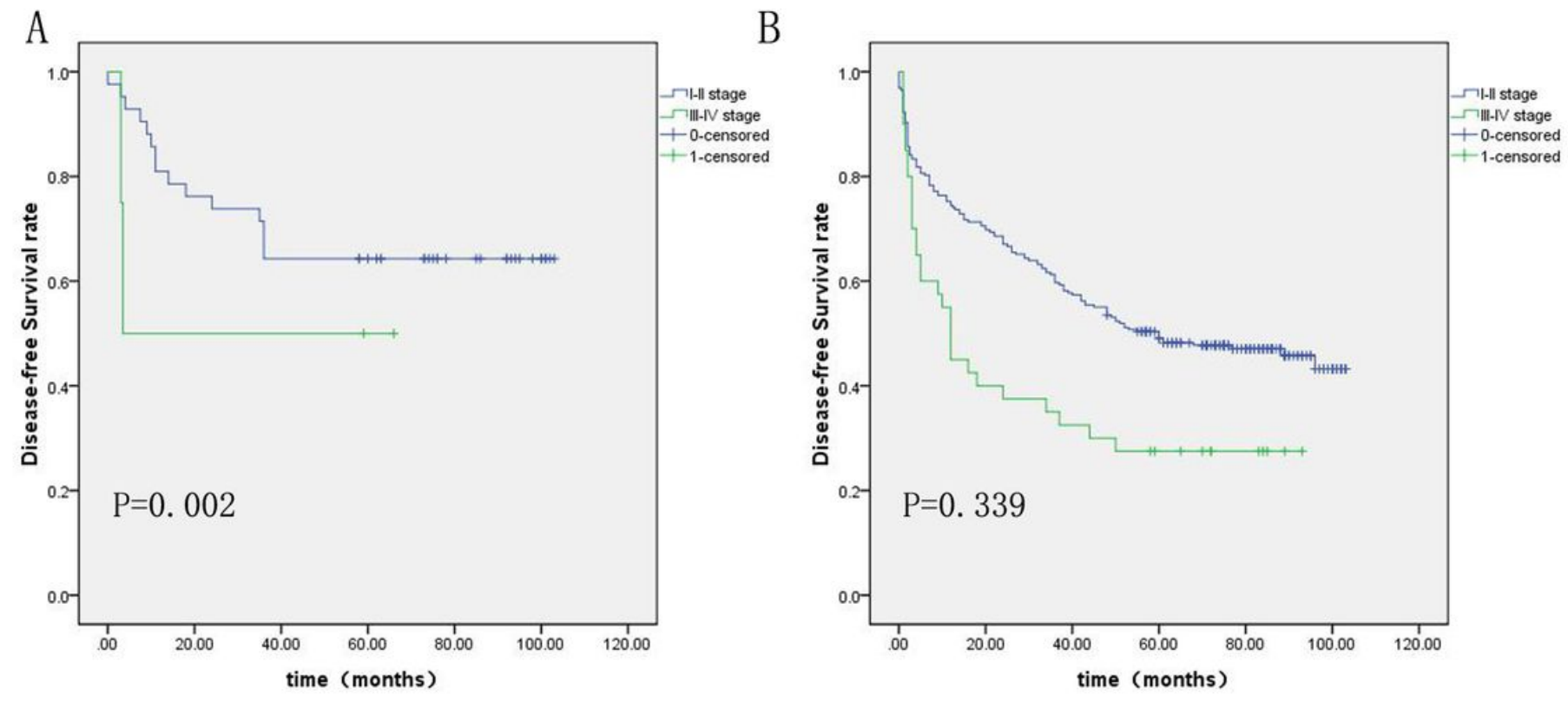

Figure 5

Kaplan-Meier curve for Disease-Free Survival according to history of hepatitis B groups. 

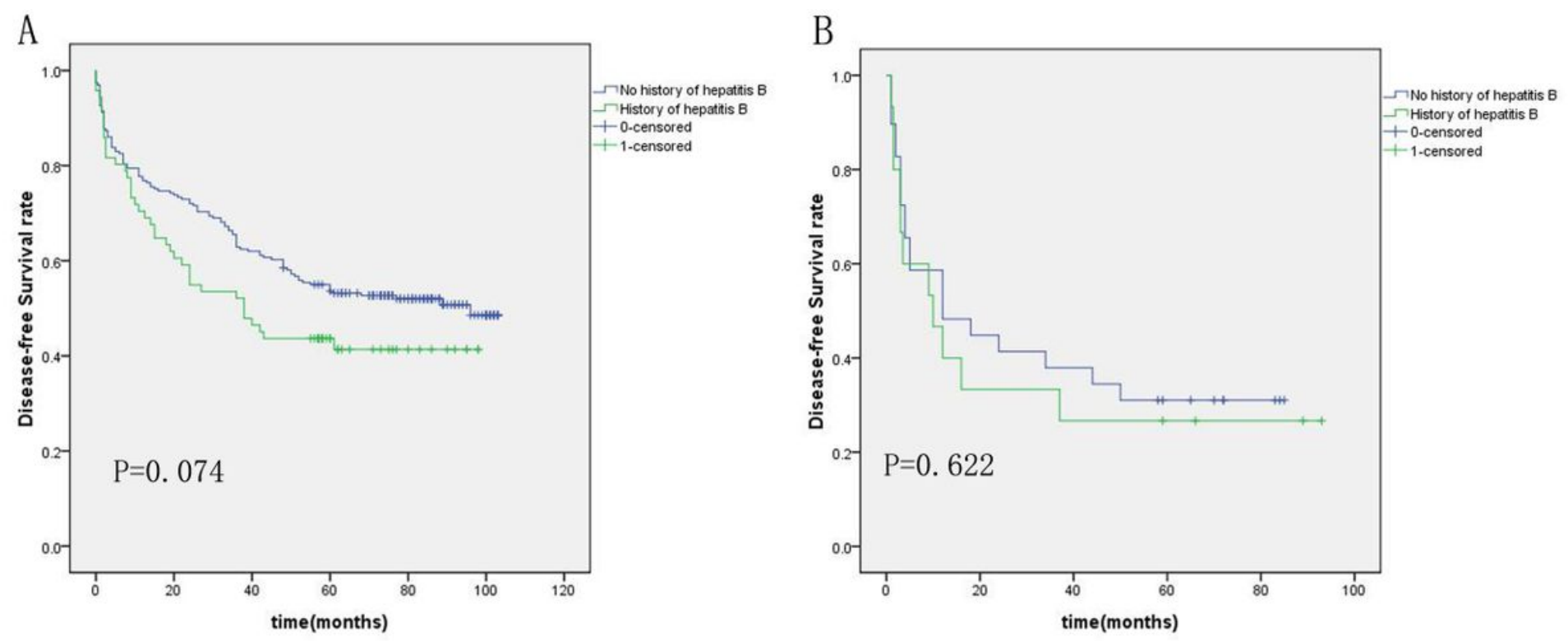

Figure 6

Kaplan-Meier curves for Disease-Free Survival in different history of hepatitis B groups based on (A) stage I- II and (B) stage III- IV. 